\title{
The design and implementation of the environment monitoring system of smart home based on EnOcean technology
}

\author{
Peng Dong ${ }^{1, a}$, Yunliang Wang ${ }^{2, b^{*}}$ \\ ${ }^{1}$ School of Electrical Engineering, Tianjin University of Technology, Tianjin, China \\ ${ }^{2}$ Tianjin Key Laboratory for Control Theory \& Applications in Complicated Systems \\ Tianjin University of Technology, Tianjin, China

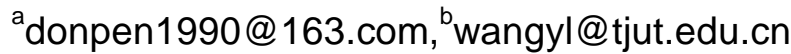

\begin{abstract}
Keywords: Environment Monitoring System; Smart Home; EnOcean Communication Technology; B/S Framework; MVC Mode

Abstract. The environment monitoring system is an important component of the smart home system. The real-time collection of data related to the temperature, humidity, luminosity plays a vital role in improving people's household living environment. To solve the problems of complex routing, low intelligence and high energy consumption of the traditional environment monitoring system, this paper designs and achieves the environment monitoring system of smart home system based on EnOcean technology. This system takes advantage of EnOcean wireless communication technique. Data related to the temperature, humidity and luminosity are collected by each acquisition unit of EnOcean and transmitted to the controller of the smart home. Those data are then transmitted to the server by controller through Wi-Fi and finally displayed real-time monitoring in browser through PC. The software of this system adopts the B/S framework and takes use of Tomcat service and the classic MVC mode to conduct the overall design.
\end{abstract}

\section{Introduction}

All With the improvement of living standards, people come to have a higher requirement for the quality of their living environment. Smart home environment monitoring plays a vital and efficient role in improving people's living quality and environment [1]. Currently, there are two categories when it comes to the researches about the environment monitoring system: the first one is wired, about which Guidong Wang and some others design in-door environment monitoring system based on CAN bus network [2], Chunying Liang and some others design temperature and humidity monitoring system in distributed greenhouse environment [3]. The second one is wireless, about which Jian Dai, Zhicai Shi and some others design environment monitoring and acquisition system based on GSM and ZigBee [4], Guangwei Sun, Xiaotian Yang and some others design wearable parameter acquisition system in smart environment based on Bluetooth technology [5]. However, the above traditional environment monitoring systems have such disadvantages as complex routing, low intelligence and high energy consumption. Thus, the author develops a new smart home environment monitoring system, which enjoys simple routing, high intelligence and low energy consumption. This system can conduct data acquisition of temperature, humidity and luminosity, and then use the EnOcean wireless communication technology to transmit the collected data to the server. Then it conducts real-time monitoring through the browser.

\section{The EnOcean wireless technology}

Based on energy harvesting, EnOcean is a kind of short-distance wireless communication technology with ultra-low power consumption, which is mainly applied in the automation of buildings. There are three advantages of this technology: chipsets with ultra-low power consumption, energy harvesting and wireless communication with high quality. EnOcean technology can collect the ultra-low energy that exist in the nature, such as light, heat, radio wave, vibration, body movement, etc. The weak energy adopted in nature can supply the wireless communication module with ultra-low power 
consumption of EnOcean, which can achieve non-battery and maintenance-free. In addition, EnOcean has very simple protocol stack, the hand-grip-free communication mechanism of which enjoys lower power consumption and higher efficiency compared with other wireless communication technology [6].

The network architecture of EnOcean mainly includes: physical layer, data link layer, network layer and application layer, the first three layers of which are based on International Standard ISO/IEC 14543-3-10 and the last layer is developed by the EnOcean Alliance.

The definition of ISO/IEC 14543-3-10:

(1)Physical layer. It adopts the radio-frequency of $315 \mathrm{MHz}$ or $868.3 \mathrm{MHz}$, the modulation method of ASK. Its effective transmission speed is $125 \mathrm{Kbit} / \mathrm{s}$ and the standard communication distance is $30 \mathrm{~m}$ indoor and $300 \mathrm{~m}$ outdoor.

(2)Data link layer. It is developed to manage the time mechanism of sub-message and detect data integrity. To ensure the reliability of transmission, it'll adopt "monitoring before sending" mechanism when sending. Besides, every message will be sent three times on the basis of a specific time algorithm. (3)Network layer. It is responsible for data packet transformation and forwarding, and the orientation of potential data packet.

Every EnOcean equipment has the only 32-bit hardware address, which is used in the equipment identification during the process of communication [7].

\section{The system structure}

This system adopts wireless data collection, which not only enjoys nice appearance, but also can effectively reduce the laying of extra cable. In this way, it cuts the cost and is the preferred option in the environment monitoring of smart home. However, in the design of environment monitoring system, except for the performance of wireless communication, energy consumption and energy resources should also be considered. This scheme introduces EnOcean wireless communication technology with ultra-low power consumption to be the basic communication way in data collection.

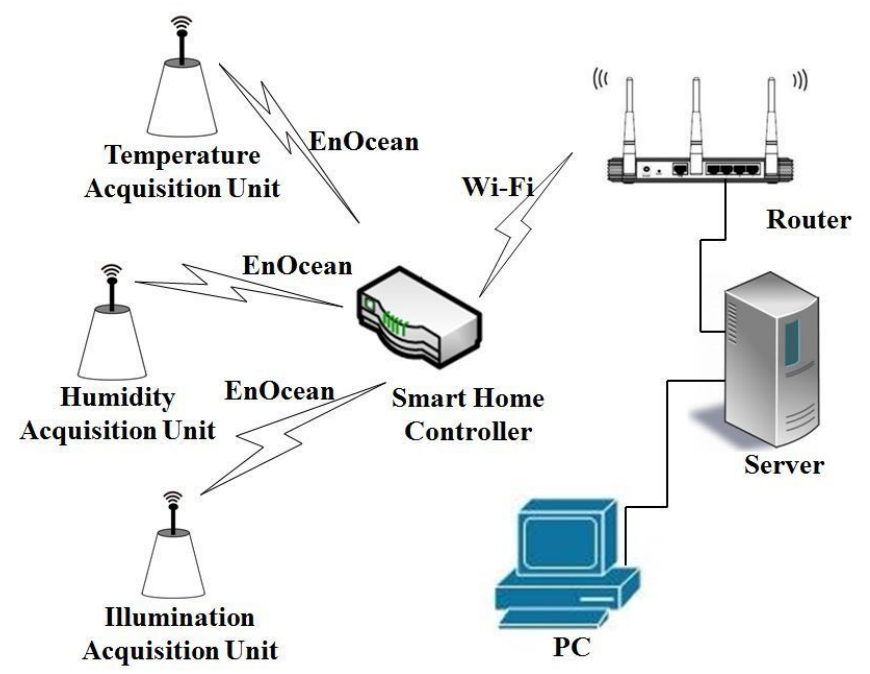

Figure 1: The topological structure of the environment monitoring system

Figure 1 displays the topological structure of the environment monitoring system of smart home based on EnOcean. In this system, data collection sensors of temperature, humidity and illumination are put in different places indoor and the collected data are then dealt with by the micro-controllers in this system. Then the EnOcean wireless communication module will send those data to the controller of smart home and the controller will send data to the router after receiving them through Wi-Fi. Next, the router uploads those data to the server through network cable and at last the browser in PC displays those data. 


\section{The design of hardware}

(1)The collection unit of EnOcean. The collection unit of EnOcean adopts energy collection technology to acquire needed electric energy. As shows in Figure 2, its components mainly include: energy collection module, energy management module, sensor module, micro-controller module, RF module and wireless module.

In the sensor module, it converts the temperature, humidity and illumination of indoor environment into electric signal and inputs the collected electric signal in the microcontroller. The microcontroller conducts filting for colleted data and then sends those data to the router, finally to the server. In the module of energy convert, the collect energy from environment is converted into electric energy and then this module will provide those stored energy to supply the work of other modules through the module of energy management [8].

(2)Controller of smart home. Simply speaking, smart home includes three kinds of equipment: home equipment, controller and server of smart home. The controller of smart home connects the home equipment and server of smart home through wireless communication, which plays a role of data bridge.

The controller of smart home adopted by this system mainly includes: microcontroller module, EnOcean communication module, Wi-Fi communication module, power module, clock and reset module. As shows in Figure 3.

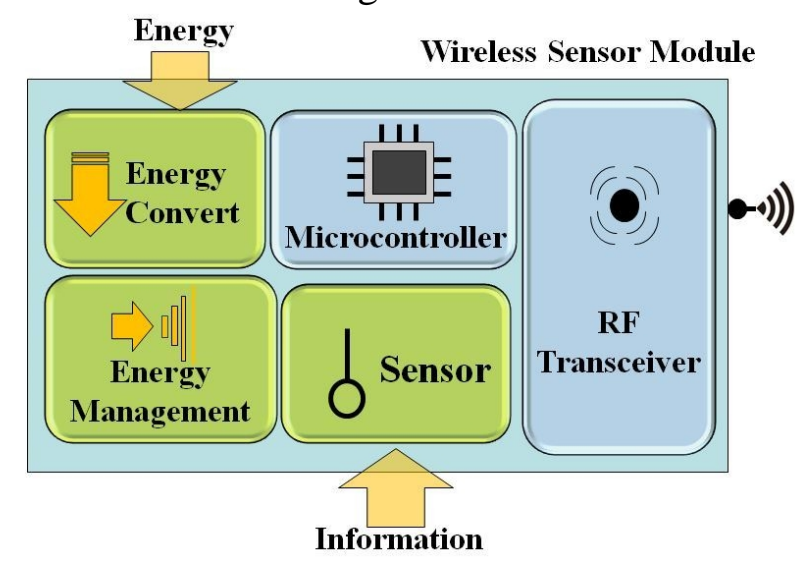

Figure 2: The hardware structure chart of the collection unit of EnOcean

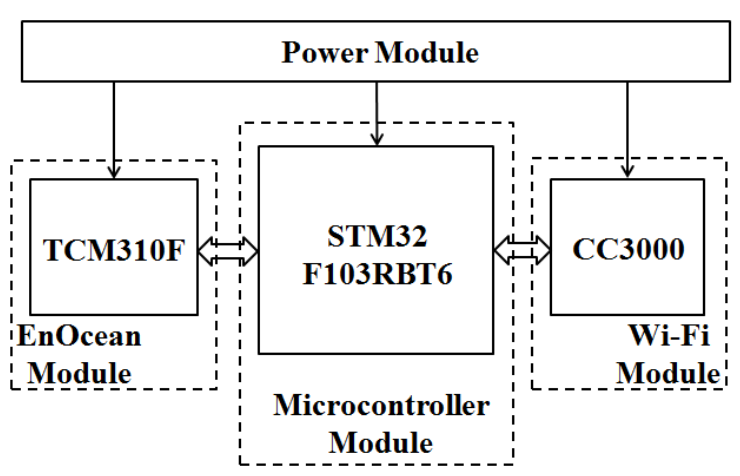

Figure 3: The structure chart of smart home controller

This controller adopts STM32F103RBT6 microcontroller, the clock frequency of which reaches $72 \mathrm{MHz}$ and can achieve high-end operation. It embeds $128 \mathrm{~KB}$ Flash program storage, enjoys rich peripheral, UART, SPI and other serial interface, GPIO with the highest transition rate $180 \mathrm{MHz}$. Most importantly, it has double 12-bit ADC with the fastest switching speed of $1 \mu \mathrm{s}$. ADC of high performance is very suitable for fast collection and dispose of data. This is also the very reason that this system chooses it to be the key controller.

EnOcean communication module adopts the integration module TCM310 of EnOcean Company. TCM310 has a two-way serial communication interface and a radio frequency communication interface, the wireless communication frequency of which is $868 \mathrm{MHz}$. Inside the TCM310, it integrates the clock module, antenna module and a radio frequency SOC chip EO3000I. EO3000I is a kind of radio frequency SOC with low energy consumption, which based on a 8051 kernel of $16 \mathrm{MHz}$ and a RF transceiver of 315/868MHz. Inside it, there are Flash of 32KB and RAM of 2KB, and 10-bit A-D and 8-bit D-A interface. EO3000I enjoys ultra-low energy consumption and the electric current of which is only $0.2 \mu \mathrm{A}$ in the mode of dormancy.

Wi-Fi communication module adopts Wi-Fi embedded chip CC3000 of TI Company to provide Internet linking ability for electronic equipment. This chip contains intact TCP/IP protocol stack and Wi-Fi drive, supports standard Socket programming and enjoys the function of wireless card IEEE $802.11 \mathrm{~b} / \mathrm{g}$. In the working mode of IEEE $802.11 \mathrm{~b} / \mathrm{g}$, the energy consumption of transmission is $190 \mathrm{~mA}$ and the energy consumption of acceptance is $92 \mathrm{~mA}$. 


\section{The design of the software based on $\mathrm{B} / \mathrm{S}$ framework}

$\mathrm{B} / \mathrm{S}$ framework is on the basis of the development mode of Internet browser and system server.

(1)The built of server. There are many kinds of B/S framework. This system uses Tomcat serer, which is developed and maintained by Apache open source group and can support JSP and Servlet to develop. Myeclipse can integrates Tomcat, after this it will debug to publish WEB projects and deploy projects directly into server. Thus the server of B/S framework is built.

(2)The development of dynamic web page. This design adopts JSP ( Java Server Pages ) technology, The very reason is that it enjoys the following advantages: a. It can operate in any Web or application server; b. It can do fast development and testing; c. It divides the logic of application programs and page display; d. It simplifies the process of development based on Web interactive applications.

(3)The operation mechanism of dynamic web page of B/S framework. In the process of designing environment monitoring system of $\mathrm{B} / \mathrm{S}$ framework, one should firstly get to know the operation mechanism of B/S framework, as shows in Figure 4.

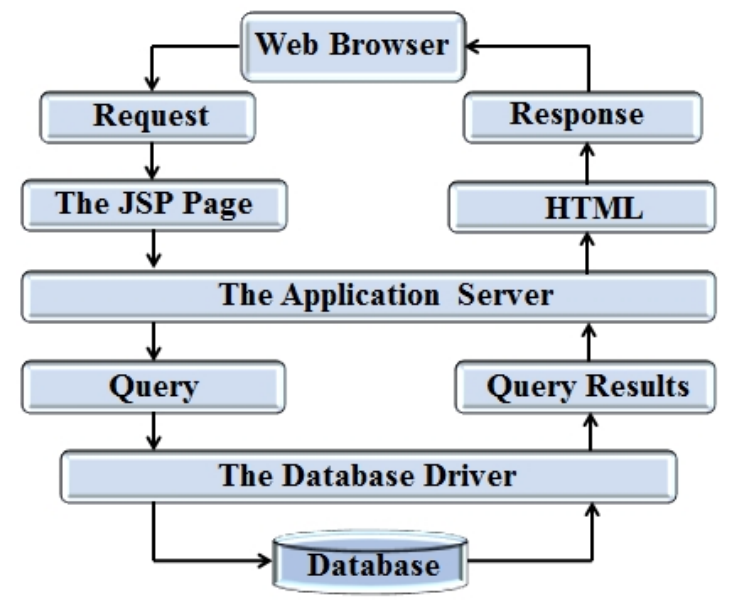

Figure 4: The operation mechanism of $\mathrm{B} / \mathrm{S}$ framework

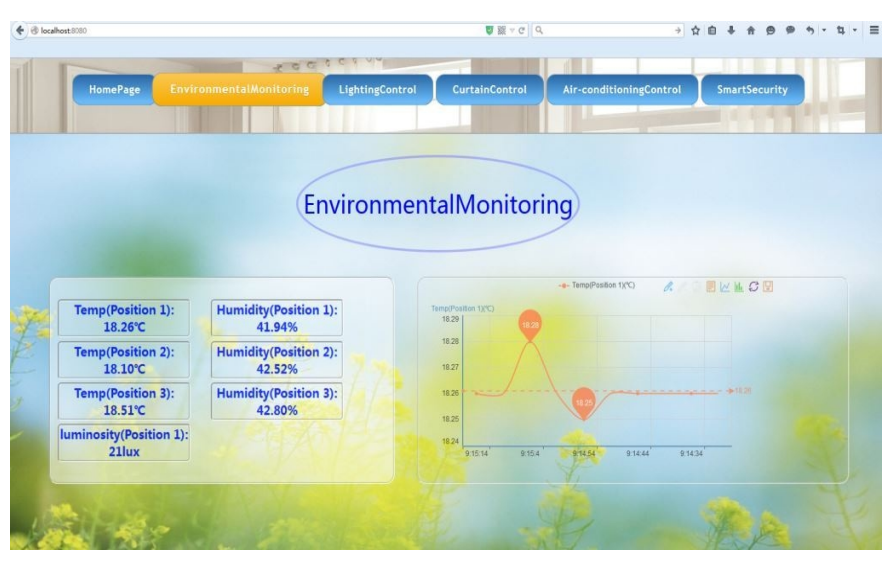

Figure 5: The monitoring page

(4) Conclusions. The software framework of this system is B/S framework. The server side adopts Tomcat server and takes advantage of classic MVC design model to conduct complete design.

This system adopts SQLite database to store data, uses JSP to display front data, uses JQuery to operate the scripting language and page layout, and designs the background program by the light weight framework Jfinal.

\section{The implementation of environment monitoring system}

(1) Monitoring page. As shows in Figure 5, the software system designed by using B/S framework displays the collected data of temperature, humidity and illumination on the browser. Enter address in the address bar of Firefox browser: localhost:8080, one can enter into the environment monitoring page, which mainly includes such three parts as the headline on the top, real-time data list on the left and real-time data curve chart on the right.

To make the collected data more accurate, it adopts multi-point collection method in the process of system design. Three positions position 1, position 2, and position 3 of the room are chosen to install the sensors. Three sensors, including one temperature sensor, one humidity sensor and one illumination sensor, are installed on position 1. Two sensors, including one temperature sensor and one humidity sensor, are installed on position 2 and position 3.

(2) The display of monitoring data. In the real-time data figure, the horizontal axis denotes time and the vertical axis denotes the gathered data. The numerical indicator of the horizontal time axis moves to the right direction and updates with the real-time. The gathered data for temperature, humidity and illumination are rounded to two decimal digits. 
Take the example of the collected data on the position 1, Figure 6 shows the real-time data curve chart of temperature on the position 1 . Figure 7 shows the real-time data curve chart of humidity on the position 1. Figure 8 shows the real-time data curve chart of illumination on the position 1.

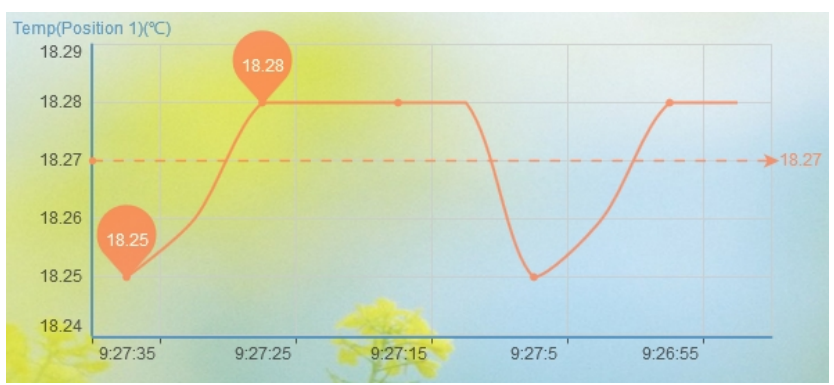

Figure 6: The real-time data curve chart of temperature on the position 1

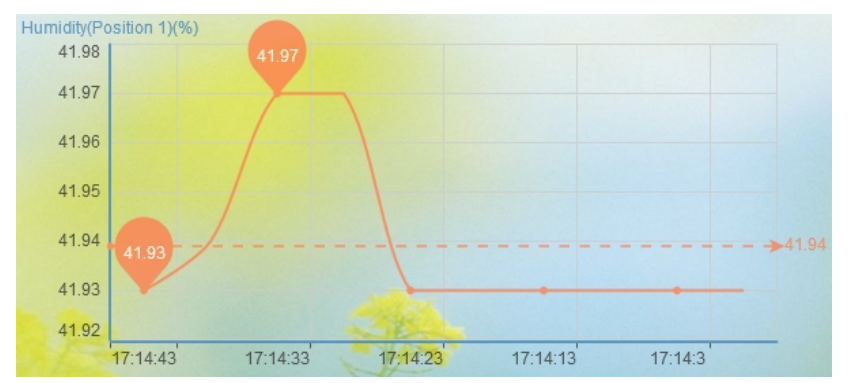

Figure 7: The real-time data curve chart of humidity on the position 1

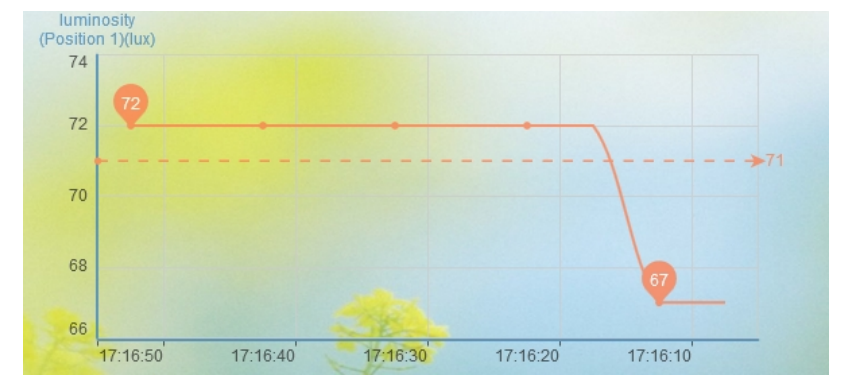

Figure 8: The real-time data curve chart of illumination on the position 1

\section{Conclusions}

This design implements the environment monitoring system of smart home based on EnOcean technology. This system takes advantage of EnOcean wireless communication technique. Data related to the temperature, humidity and luminosity are collected by each acquisition unit of EnOcean and transmitted to the controller of the smart home. Those data are then transmitted to the server by controller through $\mathrm{Wi}-\mathrm{Fi}$ and finally displayed real-time monitoring in browser through PC. The software of this system adopts the B/S framework and takes use of Tomcat service and the classic MVC mode to conduct the overall design.

Compared with the traditional environment monitoring system, this system enjoys the following advantages: the system has perfect functions and data collection of temperature, humidity and illumination is real-time; second, this system enjoys visualized display, simple operation, less wiring and is convenient to maintain and improve, which largely saves resources and energy; at last, according to the actual application needs and on the basis of traditional environment monitoring, this system takes advantage of Web browser and EnOcean wireless collection module to improve the intelligence of the system and enhance the practicability of the system, which extends a wide application prospect.

\section{References}

[1] Li, Lv; Jie, Luo. Source: Computer and Modernization, 2007, 11: 18-20+23.

[2] Guidong, Wang; Yuedong, Chen; Shaolei, Song. Source: Journal of Nanyang Institute of Technology, 2003, 06: 5-7.

[3] Chunying, Liang; Yixin, Sun; Xi, Wang. Source: Journal of Shenyang Institute of Engineering (Natural Science), 2010.03: 238-240.

[4] Jian, Dai; Zhicai, Shi;Fei, Wu; Changzhi, Wang; Feng, Jiang. Source:Automation Instrumentation, 2015, 09: 38-42.

[5] Guangwei, Sun; Xiaotian, Yang. Source: Journal of Changchun University, 2015, 04:1-4. 
[6] Li, Luo. Source: Computer\&Telecommunication, 2011,07: 5-7.

[7] Guang, Chen; Xiaohui, Li; Bing, Zhao; Xiaobing, Liang; Source: Electrotechnical Application, 2015, 02: 26-29.

[8] Gang, Tang; The research of Moving Target Location Based on EnOcean Technology[D], Chendu University of Technology,2011. 